\title{
XII. An easy method for adjusting the collimator of a spectroscope
}

\author{
Arthur Schuster Ph.D. F.R.A.S.
}

To cite this article: Arthur Schuster Ph.D. F.R.A.S. (1879) XII. An easy method for adjusting the collimator of a spectroscope , Philosophical Magazine Series 5, 7:41, 95-98, DOI: $10.1080 / 14786447908639565$

To link to this article: http://dx.doi.org/10.1080/14786447908639565

曲 Published online: 13 May 2009.

Submit your article to this journal $[\pi$

Џ Article views: 2

Q View related articles $₫$ 
electricity, and therefore exerts a more powerful attraction upon the positive than the negative. (On the different behaviours of positive and negative electricities, compare also G. Wiedemann and R. Rühlmann.)

If these experiments show that the passage of electricity from one particle to another takes place in various ways in different substances, and that, independently of the total temperature of the mixture, oniy certain particles are rendered luminous by the electric spark, we may further have to take into consideration that the passage of electricity from atom to atom is capable of producing oscillatory motions of their ather envelopes, yet without augmenting in corresponding measure the vis viva of the progressive motion of all the particles, as would be necessary according to the theory of gases. We find an analogy to this in the augmentation of the interior motion in a molecule without a corresponding increase of the oscillatory motion of the whole when non-luminous discharges pass through different gases: the latter undergo decompositions which otherwise would only be produced by considerable elevations of temperature. Similarly, in the phenomena of fluorescence, by the incident xther-vibrations the vis viva of the translatory motions is, for certain vibrations, increased in a way that corresponds to a direct augmentation of the progressive motion by heating to $500^{\circ}$ and more.

In order to put these assumptions to the proof, I havealready commenced a series of experiments, in which the temperatures of the discharges in Geissler tubes under various conditions are determined.

Leipzig, August 1878.

XII. An easy Method for Adjusting the Collimutor of a Spectroscope. By Arthul Schuster, Ph.D., F.R.A.S.*'

THE ordinary method for adjusting the collimator of a 1 spectroscope for parallel rays is only applicable to the mean rays of an achromatic combination. At the extreme ends of the spectrum a readjustment has to be made. If the ultra-violet rays are observed, and if the lenses are of quartz, the ordinary method cannot be used. The following method is so simple that I cannot help thinking it has often been in use ; yet I have nowhere seen it described, and I know that others, like myself, have often found a difficulty in making

* Commuicated by the Physical Society. 
the adjustment without much loss of time and with simple apparatus.

The adjustment, as the following consideration will show, can be made on each line of the spectrum without any apparatus whatever. The only requirement is that the prism should be movable.

Suppose the rays which fall on the prism to be either convergent or divergent; then, after their passage through the prism they will seem either to converge to or diverge from a point, which is the secondary focus : as the prism is turned, so as to change the first angle of incidence, the secondary focus will change. If the rays are strictly parallel, then, whatever be the position of the prism, the focus will not be altered. This, then, is a delicate test for ascertaining whether rays proceeding from the collimator are parallel or not. It remains to be shown how it can be converted into a rapid method to put the collimator into the right adjustment.

The three fundamental equations for the passage of a ray of light through a prism,

give

$$
\begin{aligned}
& \sin i=n \sin r, . \quad . \quad . \quad . \quad . \quad . \\
& \sin i^{\prime}=n \sin r^{\prime}, \quad \text {. . . . . . . } \\
& r+r^{\prime}=\alpha, \text {. . . . . . . . } \\
& \frac{d i^{\prime}}{d i}=-\frac{\cos i \cos r^{\prime}}{\cos i^{\prime} \cos r} . \quad \text {. . . . . }
\end{aligned}
$$

In these equations $i$ and $i^{\prime}$ are the angles which the ray makes with the first and second surfaces respectively on entering and leaving the prism; $r$ and $r^{\prime}$ the two corresponding angles of refraction, and $\alpha$ the angle of the prism. The righthand side of equation (4) will, as a little reflection will show, steadily decrease when $i$ is increased from 0 to $\frac{\pi}{2}$. This shows that the greater the first angle of incidence the more nearly parallel are the rays. The following system of consecutive approximation will therefore give the desired result.

Suppose the collimator is out of adjustment: move the telescope slightly out of position of minimum deviation; then two positions of the prism exist which will bring the desired ray into the middle of the field. Call the position in which the first angle of incidence is greatest $A$, the other $B$.

1. Put the prism into the position $A$, and focus the telescope 
until the line in question, either dark or bright, is distinctly seen.

2. Move the prism into position $\mathrm{B}$, and focus the collimator until the same line is distinctly seen.

3. Repeat the operation, always focusing the telescope when the prism is in position $A$, and the collimator when the prism is in position B. After three or four trials no change of focus is required; both collimator and telescope will then be adjusted for parallel rays. I find that it is by no means necessary to work much out of the position of minimum deviation in order to gain a delicate adjustment. If the adjustment is made in the centre of the field, then I usually put the telescope into such a position that the line, when the prism is placed at maximum deviation, should just be out of the field of view; this gives quite a sufficient change of focus if the rays are not parallel on entering the prism.

The following measurements, which were purposely mado without special care, will show the accuracy of which the method is capable. The sliding tube of the collimator was divided into millimetres. Two different adjustments for the sodiumline, made in the way described above, gave the readings 5.0 and $4 \cdot 0$. The prism was now turned round so as to deflect the ray to the other side. Two adjustments now gave $4 \cdot 1$ and $5 \cdot 0$. The mean of the four readings is $4 \cdot 5$. The adjustment was then made according to the well-known method of first focusing the telescope on a distant object and focusing the collimator to the telescope afterwards : the reading was $4 \cdot 2$. As the focal length of the collimator was 300 millimetres, the two results differ only by a thousandth part of the focal length. Whether this difference is due to errors of observation, or whether it is produced by a difference in the focus of the yellow rays and the mean visible rays, I cannot say; but I believe, with a little precaution, the method can be adapted to the study of the achromatism of a lens.

I have assumed that the faces of the prism are perfectly plane. Practically it is difficult to get a prism in which this condition is accurately fulfilled; and it may be questioned whether the curvature of the prism may not seriously interfere with the accuracy of the method. To this I reply:-

1. That a prism which is known to be good may always be set aside to do this work.

2. That the reason of having the rays strictly parnllel on entering the prism is based on the supposition that the faces of the prism are plane. It is by no means evident that parallel rays will give the best definition when the faces of the prism are curved. 
3. That the change in the adjustment of the collimator introduced by the curvature of the prism is very small. One prism, which I know to be exceptionally bad, gave a difference of a half per cent. in the focal length of the collimator. It is not the change of focus introduced by the curvatare of the prism which makes the method inaccurate when the prism is bad, but the difference in the change of focus in the two positions of the prism. This is one of the reasons why it is better to take the two positions of the prism not too far away from minimum deviation. The small displacement of the prism will only introduce a small variation in the focal length due to the curvature of the faces.

XIII. On the Morphological Laws of the Configurations formed by Magnets floating vertically and suljected to the Attraction of a superposed Magnet; with Notes on some of the Phenomena in Molecular Structure which these experiments may serve to explain and illustrate. By ALFRED M. MAYER*.

$\mathrm{I}^{\mathrm{N}}$ $\mathrm{N}$ the May Number (1878) of this Journal (page 397 of vol. v.), a short note was published on my experiments with magnets floating vertically and subjected to the attraction of a superposed magnet. The object of this paper is to present accurate diagrams of the configurations formed by the floating magnets, and to give the laws ruling these configurations, with some notices of the peculiarities of these forms. At the same time I will show how neatly these experiments illustrate several phenomena in the molecular structure of matter.

The Diagrams.--'These diagrams show the configurations formed by numbers of magnets extending from two to twenty. They were obtained as follows:- The number of needles forming a configuration were floated in a bowl filled to its brim with water. The eye-ends of the needles, which protruded a short distance beyond the tops of the corks, were of S. polarity. A cylindrical magnet, 38 centims. long and 15 millims. in diameter, was clamped in a vertical position, with its $N$. end at the constant distance of 60 millims. above the tip of the needle, which floated in the line of the axis of the magnet. I tipped the ends of the needles with printer's ink; and when the configuration had formed and was stationary, I brought down upon the needles a piece of flat cardboard, and thus obtained prints from nature. Around each of the dots on the card-

* Communicated by the Author. 